\title{
Effect of yoga on pulse rate variability measured from a venous pressure waveform
}

\author{
David Hernando, Mimma Nardelli, Kyle Hocking, Jesús Lázaro, Bret Alvis, Eduardo Gil, Enzo P. Scilingo, \\ Daniel R. Brophy, Gaetano Valenza, Pablo Laguna, Colleen Brophy and Raquel Bailón
}

\begin{abstract}
The benefits of yoga have been studied in different fields, from chronic health conditions to mental disorders, showing that it can help to improve the overall health. In particular, it has been proven that yoga also improves the autonomic function. Heart rate variability (HRV) at rest is commonly used as a non-invasive measure of autonomic regulation of heart rate. Alternatively, pulse rate variability (PRV) has been proposed as a surrogate of HRV. VoluMetrix has developed a novel technology that captures venous waveforms via sensors on the volar aspect of the wrist, called NIVAband. This study aims to assess the effect of yoga in the autonomic nervous system by analyzing the PRV obtained from the NIVA signal. Temporal (statistics of the normal-to-normal intervals), spectral (power in low and high frequency bands) and nonlinear (lagged Poincaré Plot analysis) parameters are analyzed before and after a yoga session in 20 healthy volunteers. The PRV analysis shows an increase in parameters related to parasympathetic activity and overall variability, and a decrease in parameters related to sympathetic activity and mean heart rate. These results support the beneficial effect of yoga in autonomic nervous system, increasing the parasympathetic activity.
\end{abstract}

\section{INTRODUCTION}

Several studies have taken advantage of the peripheral venous line in hospitalized patients, used primarily to allow fluids and medications to be given directly into the circulatory system, to derive patients' hemodynamic information.

\footnotetext{
*This work was supported by Centro de Investigación Biomédica en Red-Bioingeniería, Biomateriales y Nanomedicina (CIBER-BBN) through Instituto de Salud Carlos III, by the Ministerio de Economía, Industria y Competitividad, Gobierno de España, European Regional Development Fund (DPI2016-75458-R and RTI2018-097723-B-I00), by Aragón Government through Grupo de Referencia BSICoS (T39_17R), by Aragón Institute of Engineering Research (I3A), IIS Aragón and European Social Fund (EU). This project has received funding from the European Union's Framework Programme for Research and Innovation Horizon 2020 (20142020) under the Marie Sklodowska-Curie Grant Agreement No. 745755. The computation was performed by the ICTS "NANBIOSIS", more specifically by the High Performance Computing Unit of the CIBER-BBN at the University of Zaragoza.

D. Hernando (corresponding author), J. Lázaro, E. Gil, P. Laguna and R. Bailón are with CIBER-BBN, Spain and BSICoS Group, I3A, IIS Aragón, University of Zaragoza, Spain dhernand at unizar.es. J. Lázaro is with the Department of Biomedical Engineering, University of Connecticut, Storrs, CT 06269, USA. M. Nardelli, E.P. Scilingo and G. Valenza are with the Department of Information Engineering \& Research Centre E. Piaggio, School of Engineering, University of Pisa, Pisa, Italy. Kyle Hocking, PhD, is Founder, CEO and President of VoluMetrix and an inventor on intellectual property in the field of venous waveform analysis assigned to Vanderbilt and licensed to VoluMetrix. Colleen Brophy, MD, is Founder and CMO of VoluMetrix and an inventor on intellectual property in the field of venous waveform analysis assigned to Vanderbilt and licensed to VoluMetrix. Bret Alvis, MD, owns stock in VoluMetrix and an inventor on intellectual property in the field of venous waveform analysis assigned to Vanderbilt and licensed to VoluMetrix and is married to the COO of VoluMetrix. Daniel R. Brophy is a consultant with VoluMetrix, Nashville TN, USA.
}

For example, continuous monitoring of intravascular volume status permitted to detect early Stage 1 hemorrhage, while other physiological parameters such as heart rate, $\mathrm{SpO} 2$ or mean arterial pressure did not show any differences [1]. Also, peripheral venous signal is able to not only give the volume status of the patient, but also additional information such as the respiratory rate and pulse rate [2].

This capability to offer valuable additional information raises the interest of obtaining this signal in a non-invasive way that will allow a continuous monitoring in daily life. VoluMetrix is developing a new technology to capture venous waveforms non-invasively via sensors on the volar aspect of the wrist, called NIVAband. They propose Non-Invasive Venous waveform Analysis (NIVA) to obtain information on volume status, heart rate, and respiratory rate. In a previous work, we validated the ability of NIVAband to provide a measure of autonomic nervous system (ANS) via pulse rate variability (PRV) analysis [3]. Results showed that high frequency power was significantly higher in PRV analysis from NIVA than in heart rate variability (HRV) from ECG, suggesting that the NIVA signal may enhance measurement of parasympathetic activity.

Some authors have studied the effect of yoga on the autonomic function via HRV analysis, showing an increase in parasympathetic activity [4], [5]. In this work, we want to study the effect of yoga on PRV measured with the NIVAband. We will obtain both temporal and spectral PRV parameters before and after a yoga session in 20 volunteers. In addition, we propose the Lagged Poincaré Plot (LPP) for nonlinear analysis of PRV series. Significant differences were already found in Poincaré Plot parameters, when they were used together with time and frequency analyses of heart rate variability, to study autonomic regulation in yogic and control groups of subjects [5]. Moreover, LPP has been been proven to be reliable for ultra-short time HRV analysis [6]. This is important since NIVA signal is very sensitive to movement and other interferences, making it difficult to have clean pulse rate series long enough for spectral analysis.

\section{MATERIALS AND METHODS}

\section{A. Experimental Protocol and Acquisition Set-up}

Twenty healthy subjects agreed to participate in the study (written informed consent was obtained from each subject). The study was performed in accordance with the Vanderbilt University Medical Center and the University of AlabamaBirmingham Institutional Review Boards. There were 5 men and 15 women with an age range of (27-79, mean 52, 
median 56). The subjects reclined in savasana position with the head and lower extremities supported with blankets. Venous waveform signals were collected for 5-6 minutes, before (pre-yoga) and after (post-yoga) a 2 hour "awareness yoga" session, which is a slow restorative type of relaxation yoga. Venous waveforms were collected using a piezoelectric sensor (Mu rata, Nagaokakyo, Japan), placed on the volar aspect of the wrist overlying the venous plexus, secured with Coban elastic wrap (3M, Minneapolis, MN). The sensor was interfaced with a prototype NIVA device (which contains an amplifier, microcontroller, and flash memory, VoluMetrix, Nashville, TN). The data was transferred to a computer as .txt files using a USB cable and analyzed as described below.

\section{B. Preprocessing and Pulse Detections}

The NIVA signal is first low-pass filtered with a cutoff frequency of $5 \mathrm{~Hz}$ to remove high frequency noise. Frequencies below $0.3 \mathrm{~Hz}$ are also removed using a high-pass filter to remove low oscillations such as the baseline wander. Pulse detection is based on [7]: first, a linear-phase FIR low-pass-differentiator (LPD) filter is applied to accentuate the upslopes of the pulses; and then an adaptative threshold is applied to detect the peaks in the LPD filtered signal, which represents the points with maximum slope in the NIVA signal. These $k$-th pulse detections are denoted as $n_{k}$.

From $n_{k}$, the pulse interval series are obtained as $d(k)=$ $n_{k}-n_{k-1}$. Five recordings (two in pre-yoga and three in post-yoga) had to be discarded due to low quality signal and poor pulse detections: more than $80 \%$ of the pulse intervals in these recordings were considered not normal, i. e., the difference between that pulse interval and the previous one was higher than $150 \mathrm{~ms}$ [8]. Therefore, only 15 out of 20 subjects were included for further analysis.

Pulses in NIVA signal are sometimes masked by noise, yielding erroneous or missed pulse detections, which result in outlier (or anomalous) pulse intervals. These outliers are identifed, based on the algorithm in [9], and removed, obtaining the normal-to-normal (NN) intervals [10]. Figure 1 shows an example of pulses detection with an incidence and its correction (upper panel), which also reflects in the NN interval series (lower panel).

\section{Pulse Rate Variability Parameters}

Temporal Domain: For temporal domain analysis, PRV parameters are derived from the NN intervals [10]. Three parameters were considered: the standard deviation of the pulse intervals $(S D N N)$, the square root of the mean squared differences of successive pulse intervals $(R M S S D)$ and the mean pulse interval time $(\overline{N N})$.

Frequency Domain: For frequency domain analysis, PRV parameters are derived from the modulating signal $m(n)$, which is assumed to have information about ANS activity, and is computed and described below. The instantaneous pulse rate signal, $d_{\mathrm{PR}}(n)$, is derived from $d(k)$, following a method based on the time-varying integral pulse frequency modulation (TVIPFM) model, and resampled at $4 \mathrm{~Hz}$. This signal is high-pass filtered to remove the mean heart rate
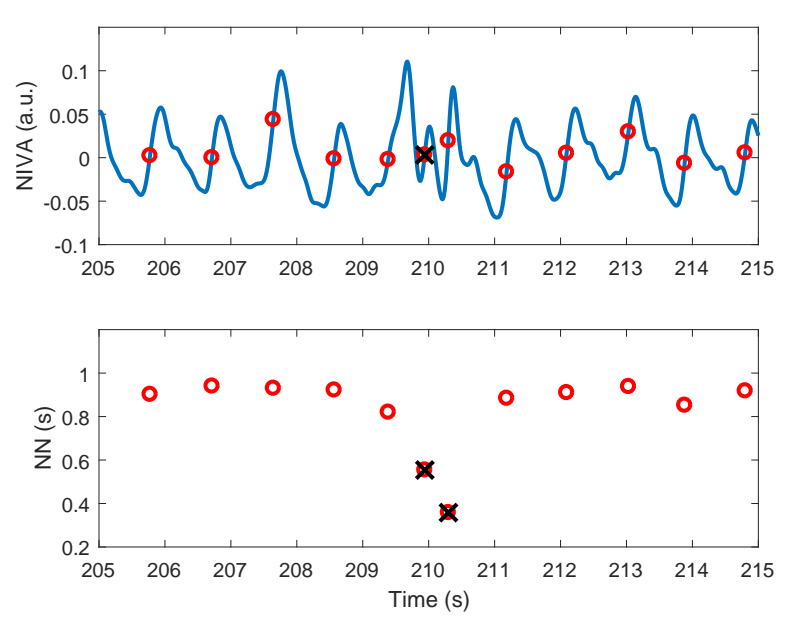

Fig. 1. Example of the NIVA signal (upper panel) with pulse detections (red circles) including an incidence (black cross). Corresponding pulse interval series (lower panel) with outlier intervals removed.

tendency $d_{\mathrm{PRM}}(n)$ (very low frequency components) and corrected to obtain the pulse rate modulating signal $m(n)$ : $m(n)=\left(d_{\mathrm{PR}}(n)-d_{\mathrm{PRM}}(n)\right) / d_{\mathrm{PRM}}(n)$ [11], [9].

Although the method in [9] takes into account the possible presence of ectopic beats, false or missed detections, if there are too many outliers following each other, the gap is too long and the estimated $m(n)$ can introduce artificial oscillations. Therefore, we do not take into account these gaps if they are longer than 3 seconds. In order to have a reliable frequency domain analysis, we only considered segments no shorter than 90 seconds without gaps longer than 3 seconds. Only 9 subjects had both pre- and post-yoga recordings suitable for frequency domain analysis.

The power spectral density (PSD) of $m(n)$ is computed using the Welch periodogram, with $60 \mathrm{~s}$ windows $10 \mathrm{~s}$ overlapped. The powers in the LF and HF bands are computed integrating the power spectrum in the corresponding bands: $P_{\mathrm{LF}}$ from 0.04 to $0.15 \mathrm{~Hz}$, and $P_{\mathrm{HF}}$ from 0.15 to $0.4 \mathrm{~Hz}$. The normalized LF power is obtained as $P_{\mathrm{LF}}=P_{\mathrm{LF}} /\left(P_{\mathrm{LF}}+P_{\mathrm{HF}}\right)$.

Lagged Poincaré Plot: The Poincaré Plot is a graphical representation of interbeat dynamics, inspired by the return map theory to describe the phase space trajectories [12]. In the standard version, the Poincare Plot is a scatterplot where each pulse interval $d(k)$ is plotted against the immediately previous pulse interval $d(k-1)$. In the lagged Poincaré Plot (LPP) technique, a lag $l$ is introduced and scatterplots are made by the points with coordinates $d(k)$ and $d(k-l)$. Previous studies investigated a range of lag values equals to $1 \leq l \leq 10$.

The most used quantitative approach to describe the shape of LPP is the ellipse fitting technique [13]. Following this methodology, the LPP is turned $45^{\circ}$ clockwise and the two standard deviations of the points around the vertical $(S D 1)$ and horizontal $(S D 2)$ axes are computed. $S D 1$ is a measure of the short-term variability of pulse interval series, whereas $S D 2$ describes long-term dynamics. In this study 
we investigated the two standard deviations, $S D 1$ and $S D 2$, together with their ratio $S D 12=S D 1 / S D 2$.

We computed the values of $S D 1, S D 2$, and $S D 12$ in $50 \%$ overlapped windows lasting $35 \mathrm{sec}$, both in pre-yoga and post-yoga NN intervals. The standard range $1 \leq l \leq$ 10 for the lag value was considered in the LPP analyses. The reliability of LPP parameters calculated using the ellipse fitting technique in $35 \mathrm{sec}$ windows was already investigated in our previous studies, through synthetic and real data [6], [14].

Considering each LPP parameter ( $S D 1, S D 2$, and $S D 12$ ) and each lag $l$, we calculated the median values among the $35 \mathrm{sec}$ windows, in the pre-yoga session and in the post-yoga session. For each subject, we obtained two median values of each LPP parameter and each lag corresponding to pre-yoga and post-yoga, respectively.

\section{Statistical analysis}

A paired Wilcoxon non-parametric statistical test was applied to all PRV parameters to study the differences between pre- and post-yoga sessions. Analysis of temporal ( $S D N N$, $R M S S D, \overline{N N})$ and LLP parameters $(S D 1, S D 2, S D 12$, for each lag) included 15 subjects, while analysis of spectral parameters $\left(P_{\mathrm{LFn}}\right.$ and $\left.P_{\mathrm{HF}}\right)$ included 9 subjects. The difference is considered to be significantly different from zero when $\mathrm{p}$ $<0.05$.

\section{RESULTS}

Figure 2 shows an example of the NN interval series for pre- and post-yoga sessions, as well as their corresponding PSD. An overall increase in power is observed after the yoga session.
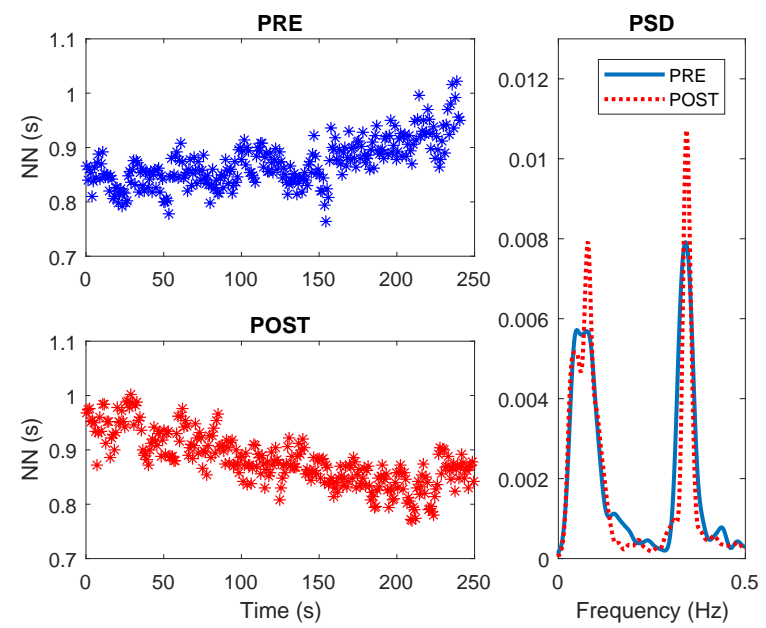

Fig. 2. Example of NN interval series for pre- and post-yoga sessions (left panels) and their corresponding power spectral densities (right panel).

Temporal PRV parameters are shown in Figure 3: there is an increase in $\overline{N N}$ (which translates to a decrease in the mean heart rate), $S D N N$ and $R M S S D$, but it is only statistically significant in $S D N N$. Spectral PRV parameters are shown in Figure 4: there is a decrease in $P_{\mathrm{LFn}}$ and an increase in $P_{\mathrm{HF}}$, but both are not significant. The only LPP parameter which was found to be statistically different was the ratio $S D 12$ (lags 5 and 7), shown in Figure 4: there is a significative increase after yoga.

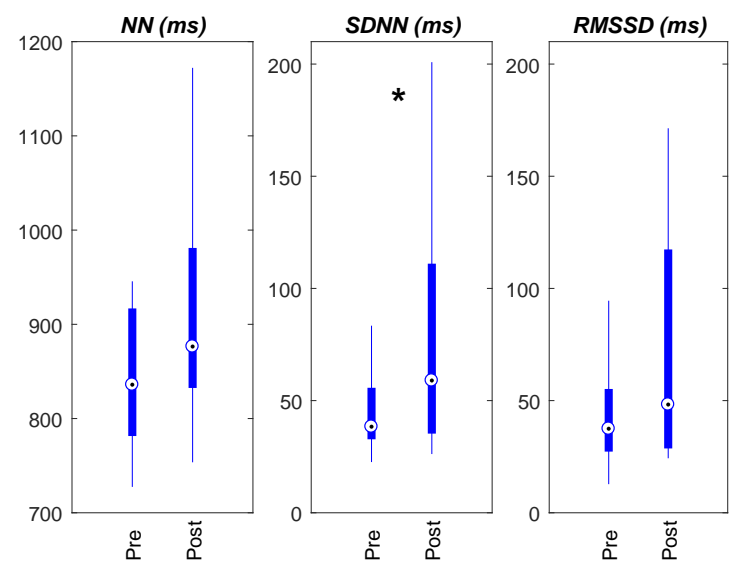

Fig. 3. Temporal PRV parameters in pre- and post-yoga recordings. * denotes significant differences $(\mathrm{p}<0.05)$.

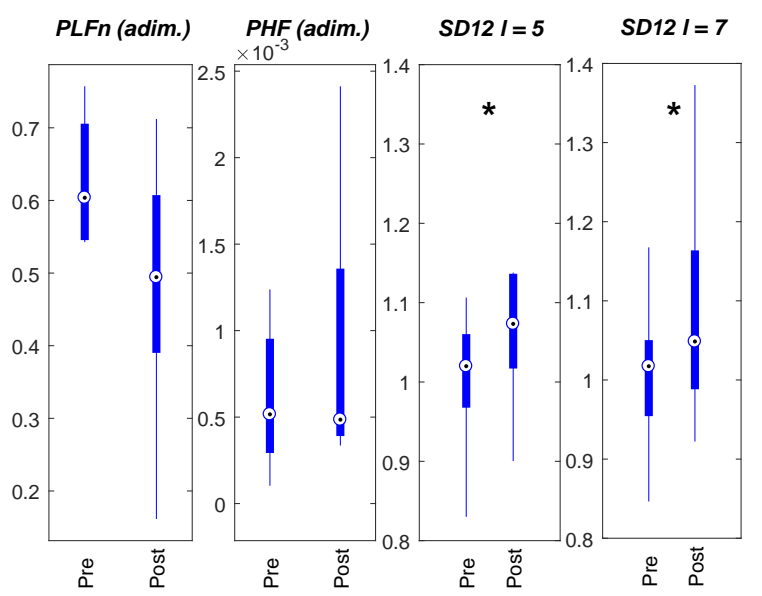

Fig. 4. Spectral and LLP (SD12, lags 5 and 7) PRV parameters in preand post-yoga recordings. $*$ denotes significant differences $(\mathrm{p}<0.05)$.

\section{DISCUSSION AND CONCLUSION}

Several works have studied the role of yoga in the overall health and a healthy lifestyle [15], [4], [5]. Also, yoga has been proposed in severe mental illnesses: it was found a reduction in general psychopathology ratings and an improvement in cognition and functioning in schizophrenic patients, and a significant benefit in reducing the severity of depressive symptoms [16]. In those studies which focus on the autonomic function, they found an increase in parasympathetic activity, a decrease in sympathetic activity and an overall increase heart rate variability [4].

Our results agree with those found in the literature. While most temporal and spectral parameters did not show 
significative differences, possibly due to the small size of data, they indeed followed the same tendencies than in other studies. We found an increase in $\overline{N N}$ (a decrease in mean heart rate) which was also observed in previous works. In most recordings, we found a tendency similar than the example shown in Figure 2: in pre-yoga, the heart rate decreases over time as the yoga session approaches, while in post-yoga the heart rate increases over time. The increase in $S D N N$ is related with an overall increase of total variability, while the increase in $R M S S D$ is related to an increased parasympathetic activity [10].

Spectral parameters also show similar results. There is a decrease in LF power (in normalized units), an increase in HF power and a decrease in the LF/HF ratio, which translates to an increase in parasympathetic activity and a decrease in both sympathetic activity and sympathovagal tone. Figure 2 also shows an increase in both LF and HF power, which may be related to the increased parasympathetic activity affecting both bands. Taking advantage of the capability of the NIVAband to estimate the respiratory rate [3], we found that it was located within the classic HF band $(0.15$ to $0.4 \mathrm{~Hz}$ ) for all recordings, and no statistical differences were found in $P_{\mathrm{HF}}$ when centering the $\mathrm{HF}$ band around respiratory rate compared to using the classic HF band. The main limitation for spectral analysis is that NIVA signal is very sensitive to movement artifacts, and only in 9 subjects we could find segments longer than $90 \mathrm{~s}$ with no outliers in the pulse interval series. We also repeated the statistical test for temporal parameters with only these 9 subjects, and the tendencies were the same, with $S D N N$ being non significative this time.

To overcome this limitation, we used other nonlinear parameters which can be computed in very short time windows, such as Lagged Poincaré Plot. Several previous studies reported the correlation between lagged $S D 1$ and HF power, revealing its strong ability to detect increase in vagal modulation [17]. According to recent literature, SD12 parameter relates to the nonlinear component of heartbeat dynamics (especially for $l=5,6$ ) and an increase of sympathetic activity was found to decrease its value [18]. Therefore, the increase in $S D 12$ found in this work suggests a decrease in sympathetic activity. We also repeated this analysis using the whole recordings instead of the $35 \mathrm{~s}$ windows, and we found similar statistical differences in SD12 with lags 4 and 5.

In summary, we have shown that the NIVAband is able to detect changes in autonomic function after a yoga session. Similar to other heart rate variability studies, we have found that yoga increases parasympathetic activity and decreases the sympathovagal balance and the mean heart rate. There is also an overall increase in the total variability. To overcome the limitations found in the NIVA signal, which make difficult to analyze PRV parameters in the frequency domain, we used temporal and non linear parameters, finding similar results than with spectral parameters. Future studies will focus on investigating the NIVA's potential enhancement of parasympathetic activity measurement compared to other
PPG devices.

\section{REFERENCES}

[1] B. Sileshi, K. Hocking, R. Boyer, F. Baudenbacher, K. Kohurst, C. Brophy, and S. Eagle, "Peripheral venous waveform analysis for detecting early hemorrhage: a pilot study," Intensive Care Med, vol. 41(6), pp. 1147-?1148, 2015.

[2] K. Hocking, B. Alvis, F. Baudenbacher, R. Boyer, C. Brophy, I. Beer, and S. Eagle, "Peripheral i.v. analysis (PIVA) of venous waveforms for volume assessment in patients undergoing haemodialysis," $\mathrm{Br} \mathrm{J}$ Anaesth., vol. 119(6), pp. 1135-1140, 2017.

[3] D. Hernando, R. McCallister, J. Lázaro, K. Hocking, E. Gil, P. Laguna, C. Brophy, and R. Bailón, "Validity of venous waveform signal for heart rate variability monitoring," in XLV International Conference on Computing in Cardiology, 2018, pp. 1-4.

[4] L. Bhaskar, C. Kharya, K. Deepak, and V. Kochupillai, "Assessment of cardiac autonomic tone following long sudarshan kriya yoga in art of living practitioners," J Altern Complement Med, vol. 23(9), pp. 705-712, 2017.

[5] S. Senthilnathan, R. Patel, M. Narayanan, G. Katholil, M. P. R. Janawadkar, T. S. Radhakrishnan, and K. K. Sharma, "An investigation on the influence of yogic methods on heart rate variability," Annals of Noninvasive Electrocardiology, vol. 24, no. 1, p. e12584, 2019.

[6] M. Nardelli, A. Greco, J. Bolea, G. Valenza, E. P. Scilingo, and R. Bailón, "Reliability of lagged poincaré plot parameters in ultrashort heart rate variability series: Application on affective sounds," IEEE journal of biomedical and health informatics, vol. 22, no. 3, pp. 741749, 2018.

[7] J. Lázaro, E. Gil, J. Vergara, and P. Laguna, "Pulse rate variability analysis for discrimination of sleep-apnea-related decreases in the amplitude fluctuations of PPG signal in children," IEEE Journal of Biomedical and Health Informatics, vol. 18(1), pp. 240-246, 2014.

[8] V. Monasterio, P. Laguna, I. Cygankiewicz, R. Vázquez, A. BayésGenís, A. Bayés de Luna, and J. Martínez, "Average T-wave alternans activity in ambulatory ECG records predicts sudden cardiac death in patients with chronic heart failure," Heart Rhythm, vol. 9(3), pp. 383389, 2012.

[9] J. Mateo and P. Laguna, "Analysis of heart rate variability in the presence of ectopic beats using the heart timing signal," IEEE Transactions on Biomedical Engineering, vol. 50, no. 3, pp. 334-343, 2003.

[10] The Task Force of ESC and NASPE, "Heart rate variability. standards of measurement, physiological interpretation, and clinical use," Eur. Heart J., vol. 17, pp. 354-381, 1996.

[11] R. Bailón, G. Laouini, C. Grao, M. Orini, P. Laguna, and O. Meste, "The integral pulse frequency modulation with time-varying threshold: application to heart rate variability analysis during exercise stress testing," IEEE Trans Biomed Eng, vol. 58(3), pp. 642-652, 2011.

[12] F. Marciano, M. Migaux, D. Acanfora, G. Furgi, and F. Rengo, "Quantification of poincare'maps for the evaluation of heart rate variability," in Computers in Cardiology 1994. IEEE, 1994, pp. 577580.

[13] M. P. Tulppo, T. Makikallio, T. Takala, T. Seppanen, and H. V. Huikuri, "Quantitative beat-to-beat analysis of heart rate dynamics during exercise," American journal of physiology-heart and circulatory physiology, vol. 271, no. 1, pp. H244-H252, 1996.

[14] M. Nardelli, A. Greco, J. Bolea, G. Valenza, E. P. Scilingo, and R. Bailón, "Investigation of lagged poincaré plot reliability in ultrashort synthetic and experimental heart rate variability series," in 2017 39th Annual International Conference of the IEEE Engineering in Medicine and Biology Society (EMBC). IEEE, 2017, pp. 2329-2332.

[15] K. Yamamoto-Morimoto, S. Horibe, R. Takao, and K. Anami, "Positive effects of yoga on physical and respiratory functions in healthy inactive middle-aged people," Int J Yoga, vol. 12(1), pp. 62-67, 2019.

[16] G. Sathyanarayanan, A. Vengadavaradan, and B. Bharadwaj, "Role of yoga and mindfulness in severe mental illnesses: A narrative review," Int J Yoga, vol. 12(1), pp. 3-28, 2019.

[17] P. Contreras, R. Canetti, and E. R. Migliaro, "Correlations between frequency-domain HRV indices and lagged poincaré plot width in healthy and diabetic subjects," Physiological measurement, vol. 28, no. 1 , p. $85,2006$.

[18] B. Koichubekov, V. Riklefs, M. Sorokina, I. Korshukov, L. Turgunova, Y. Laryushina, R. Bakirova, G. Muldaeva, E. Bekov, and M. Kultenova, "Informative nature and nonlinearity of lagged poincaré plots indices in analysis of heart rate variability," Entropy, vol. 19, no. 10, p. 523, 2017. 